\title{
Measurement of hard x-ray lens wavefront aberrations using phase retrieval
}

\author{
Manuel Guizar-Sicairos, ${ }^{1, a)}$ Suresh Narayanan, ${ }^{2}$ Aaron Stein, ${ }^{3}$ Meredith Metzler, ${ }^{4}$ \\ Alec R. Sandy, ${ }^{2}$ James R. Fienup, ${ }^{1}$ and Kenneth Evans-Lutterodt ${ }^{3}$ \\ ${ }_{1}^{1}$ The Institute of Optics, University of Rochester, Rochester, New York 14627, USA \\ ${ }^{2}$ Argonne National Laboratory, Argonne, Illinois 60439, USA \\ ${ }^{3}$ Brookhaven National Laboratory, Upton, New York 11973, USA \\ ${ }^{4}$ Cornell Nanofabrication Facility, Cornell University, New York 14853, USA
}

(Received 6 November 2010; accepted 2 February 2011; published online 15 March 2011)

\begin{abstract}
Measuring the deviation of a wavefront from a sphere provides valuable feedback on lens alignment and manufacturing errors. We demonstrate that these aberrations can be accurately measured at hard $\mathrm{x}$-ray wavelengths, from far-field intensity measurements, using phase retrieval with a moveable structure in the beam path. We induce aberrations on a hard x-ray kinoform lens through deliberate misalignment and show that the reconstructed wavefronts are in good agreement with numerical simulations. Reconstructions from independent data, with the structure at different longitudinal positions and significantly separated from the beam focus, agreed with a root mean squared error of 0.006 waves. (C) 2011 American Institute of Physics. [doi:10.1063/1.3558914]
\end{abstract}

Wavefront measurements are widely used at optical wavelengths to test the quality of optical surfaces and evaluate the overall performance and alignment of complicated imaging systems. ${ }^{1}$ If accurately measured, the deviation of the wavefront from an ideal sphere provides a quantitative map of the aberrations induced by manufacturing errors and/or misalignment.

Access to an at-wavelength hard $\mathrm{x}$-ray wavefront map is very desirable for on-site interactive alignment and/or quantitative diagnosing of the performance of focusing optics. However, there are relatively few methods to accurately measure wavefront aberrations in situ at these wavelengths. An X-ray wavefront can be measured using a Hartmann test ${ }^{2}$ or an x-ray grating interferometer. ${ }^{3}$ However, because these methods measure the wavefront slope, they can have difficulties with discontinuous wavefronts, and they are not optimally suited for measuring strongly converging (or diverging) wavefronts of high numerical aperture.

Phase retrieval can be advantageous since it has relaxed hardware and calibration requirements. For x-ray diffractive imaging, Faulkner and Rodenburg ${ }^{4}$ introduced a practical diversity mechanism that significantly improved the convergence and robustness of the phase retrieval reconstruction. In their approach, the sample of interest is moved transversely to the direction of beam propagation, with some degree of overlap, and the phase of the measured diffraction patterns is jointly reconstructed.

The technique can be conversely used to retrieve the beam incident on a known moveable structure and can thus be explicitly applied to x-ray beam characterization. This is a capability that has been numerically assessed ${ }^{5}$ and experimentally demonstrated. ${ }^{6-9}$ Because this technique does not need a measurement of intensity profile of the beam near focus, it has practical advantages over previous methods. ${ }^{10}$

In this letter, we demonstrate that phase retrieval with transverse translation diversity can accurately and reliably

\footnotetext{
${ }^{a)}$ Present address: Paul Scherrer Institut, CH-5232 Villigen PSI, Switzerland. Electronic mail: manuel.guizar-sicairos@psi.ch.
}

measure the wavefront aberrations of a focusing hard x-ray lens using far-field diffraction intensity measurements only. For our experiment, we purposely introduce known misalignments to a kinoform lens and demonstrate that the recovered wavefronts are consistent with the expected behavior and in very good agreement with detailed numerical simulations. We further demonstrate that the reconstructions are accurate when the moveable structure is significantly away from focus, a capability that allows "remote" probing of the beam focus.

For easier modeling and interpretation of results, we used a single one-dimensional (1D) kinoform lens with focal length $f=96 \mathrm{~cm}$ and aperture width of $200 \mu \mathrm{m}$. The kinoform has a nominally elliptical back surface. On the (nominally flat) front surface, material corresponding to an integer number of waves of phase advancement was removed from the design to improve transmissivity across the lens aperture. The design was lithographically patterned on a standard silicon wafer and etched using a Bosch deep reactive-ion etching technique. ${ }^{11,12}$

Figure 1(a) shows the experimental arrangement carried out at the 8-ID-I beamline of Argonne National Laboratory Advanced Photon Source (ANL-APS). The lens focused the incoming $7.35 \mathrm{keV}(\lambda=0.17 \mathrm{~nm})$ coherent beam along the vertical direction to a line focus. In the path of the beam, we placed a moveable silicon structure of $9 \mu \mathrm{m}$ thickness to perturb the beam and provide the diversity in the measurements. At this wavelength, the structure provides a phase shift of $\pi$ radians and an intensity absorption of about $16 \%$. The silicon structure transverse width and height were $200 \mu \mathrm{m} \times 50 \mu \mathrm{m}$, respectively, significantly larger than the beam transverse size.

The diffraction patterns were measured at $3.45 \mathrm{~m}$ from the structure by scanning a $30 \mu \mathrm{m}$ pinhole in $10 \mu \mathrm{m}$ steps and measuring the transmitted intensity with an FMB Oxford avalanche photodiode detector (APD). Because we are characterizing a 1D (line) focus, only 1D intensity measurements are necessary. ${ }^{5,8}$ Despite this, the data collection by a scanning point detector was slow. This could be improved sig- 


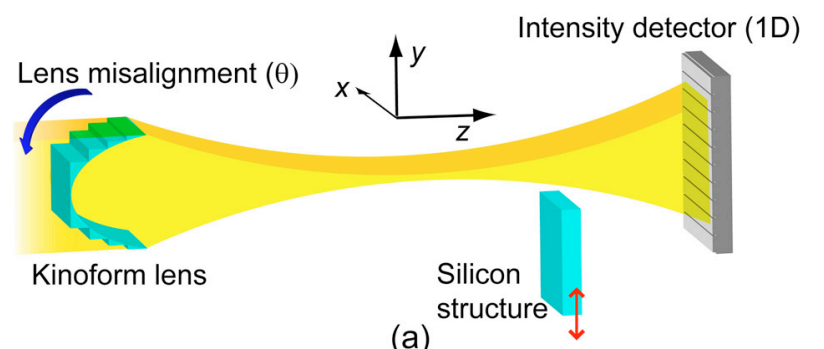

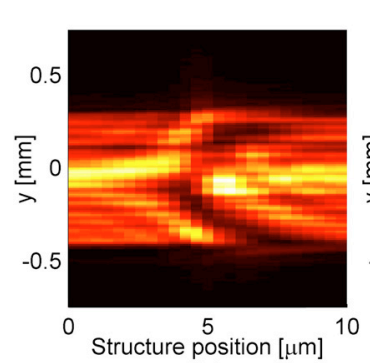

(b)

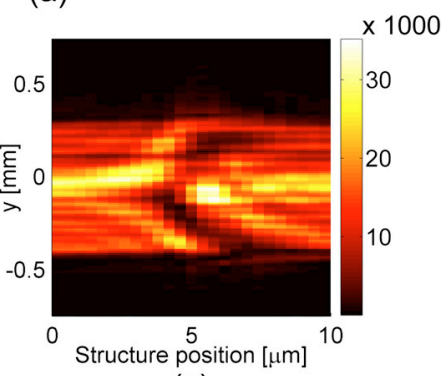

(c)
FIG. 1. (Color online) (a) Experimental arrangement. The intensity patterns were measured by scanning an APD but the measurement time can be reduced by using an intensity detector array as depicted. (b) Measured intensity vs structure position for a lens angular misalignment of $\theta=0.14^{\circ}$. (c) Computed far-field intensity vs structure position for the reconstructed beam. Color bar is in units of photon counts.

nificantly by using a good quality x-ray detector array.

A data set with 26 diffraction patterns, for a lens misalignment of $0.14^{\circ}$, is shown in Fig. 1(b). Intensity measurements are arranged vertically and the horizontal axis represents the position of the structure, which was translated in $400 \mathrm{~nm}$ steps. Analogous to the optical wire test, ${ }^{1}$ the shadow of the edge of the silicon structure maps the lens transverse ray aberrations, which are proportional to the local slope of the wavefront. Thus, the shadow profile seen in Fig. 1(b), which is approximately quadratic with position of the structure, immediately provides a qualitative indication of a thirdorder phase aberration.

The diffraction patterns in Fig. 1(b) were fed to a nonlinear optimization algorithm to reconstruct the x-ray beam and refine our initial estimate of the moveable structure transmissivity. The algorithm, described in Ref. 13, iteratively finds a beam that can reproduce the intensity measurements upon multiplication with the structure transmissivity (with a corresponding translation) and propagation to the plane of the detector. Although the $1 \mathrm{D}$ assumption of the technique is not strictly satisfied in the experiment, we have found in simulations that the diversity of measurements render the method quite robust to deviations from this approximation. ${ }^{5}$

The far-field intensity patterns numerically modeled from this reconstruction are shown in Fig. 1(c), which are in very good agreement with the measurements in Fig. 1(b). A propagation through focus of the reconstructed beam, for $\theta$ $=0.14^{\circ}$, is shown in Fig. 2(a). Similar experiments were performed where we used 26 and 31 diffraction patterns for lens angular misalignments of $\theta=-0.1^{\circ}$ and $\theta=-0.25^{\circ}$, respectively. The corresponding reconstructed beams are shown in Figs. 2(b) and 2(c).

Figure 3(a) shows the measured x-ray lens wavefront aberrations (solid lines) and their comparison with numerical simulations (dashed lines) using the beam propagation method (BPM), ${ }^{14}$ a numerical propagation method that se-
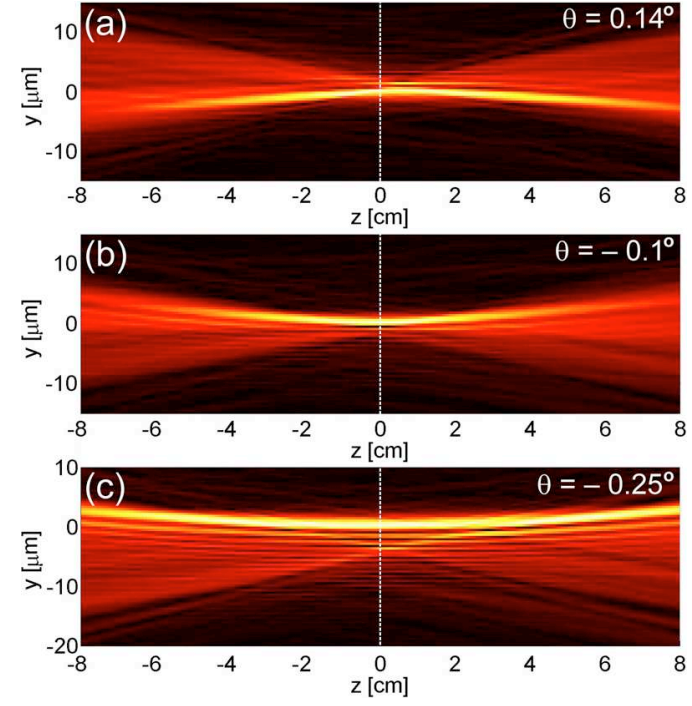

FIG. 2. (Color online) Through focus amplitude of reconstructed beams for a lens angular misalignment of (a) $\theta=0.14^{\circ}$, (b) $\theta=-0.1^{\circ}$, and (c) $\theta=-0.25^{\circ}$. White dashed lines indicate the plane of reconstruction.

quentially imparts the diffraction and refractive index effects over a small propagation distance and for which we included the effect of the rotation of a misaligned lens. At the edge of the kinoform sections, a boundary wave is formed which locally perturbs the phase and generates the spikes that can be seen on the BPM simulated wavefronts in Fig. 3(a). These phase perturbations cannot be resolved by our reconstruction, as its resolution at the plane of the lens is $9.2 \mu \mathrm{m}$, given by the geometrical projection of the detector pinhole diameter. The spikes are very localized and for our lens parameters we expect they bear a negligible effect on the quality of the focus.

Figure 3(b) shows the third order aberration coefficient (Legendre polynomial coefficient) as a function of lens misalignment angle for the simulation (solid line) and experiment (open circles). They both exhibit a linear behavior, which is expected for small misalignments. Higher order aberrations were smaller than $3 \times 10^{-4}$ waves root mean square (rms) for the simulation and 0.01 waves rms for the experimental data; the latter are presumably caused by small manufacturing errors.

To demonstrate that this technique can be used when the structure is placed a significant distance away from focus, we
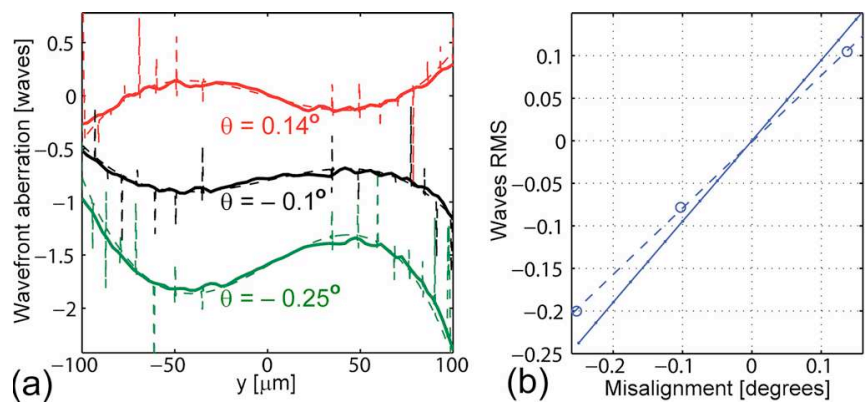

FIG. 3. (Color online) (a) Lens x-ray wavefront aberrations for different misalignment angles $\theta$. Comparison of experimental reconstructions (solid lines) vs the numerical simulations (dashed lines). (b) Third order polynomial aberration coefficient (Legendre) vs lens misalignment angle for the numerical simulations (solid line) and the experimental reconstructions (open circles). Dashed line is a linear fit to the experimental reconstructions. 

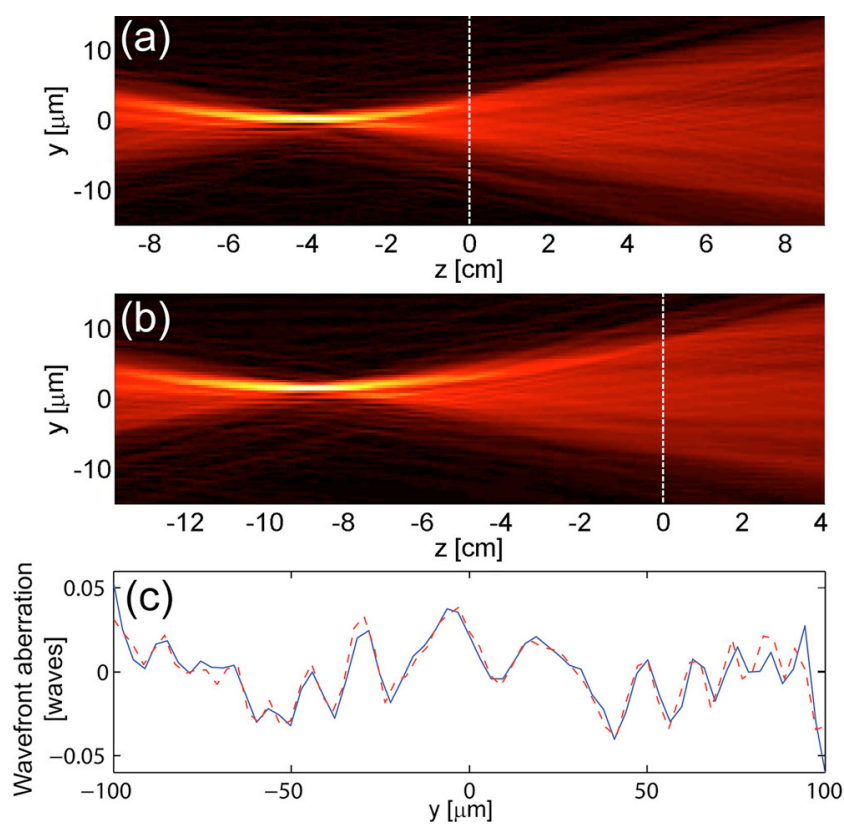

FIG. 4. (Color online) Beams reconstructed with moveable structure at (a) 4.2 and (b) $9.1 \mathrm{~cm}$ from the paraxial focus. Dashed line shows the position of the structure. (c) Residual phase aberrations of the lens. Solid and dashed lines are the wavefronts computed from the reconstructions in (a) and (b), with 0.018 and 0.019 waves rms, respectively. The two measurements agree with an error of 0.006 waves rms.

reconstructed a beam from two independent sets of measurements with 31 and 38 diffraction patterns, respectively. In both cases the structure translation step was $500 \mathrm{~nm}$ and the only modification to the setup between these two data sets was the position of the perturbing structure. In the reconstructions, shown in Fig. 4, we identified small third order aberrations of 0.029 and 0.037 waves rms for the reconstructions at $4.2 \mathrm{~cm}$ and $9.1 \mathrm{~cm}$ from focus, respectively. These aberrations arose from undesired lens misalignment. Upon removal of these aberrations we can obtain a residual phase profile that most likely arises from lens manufacturing errors. Figure 4(c) shows these residual phase aberrations which are below 0.019 waves rms; this value corresponds to $345 \mathrm{~nm}$ of silicon and is significantly smaller than $1 / 14 \sim 0.071$ waves rms, the Maréchal criterion for diffraction-limited optics. The two independently reconstructed wavefronts agree within 0.006 waves ( $1 \mathrm{pm}$ of optical path difference) rms.

We have experimentally demonstrated the capability of measuring the wavefront aberrations of hard x-ray focusing lens in situ using phase retrieval with a transversely translated structure, a technique that requires no knowledge about the focusing optics. In the experiment, we introduced aberrations by deliberately rotating a kinoform lens out of alignment and found that the reconstructed wavefronts followed the expected trend and agreed with numerical simulations. Reconstructions of comparable quality to the ones presented in this paper were obtained on-site and used as feedback for the experimental procedure.

Additionally, we compare reconstructions of the same $\mathrm{x}$-ray beam from two independent data sets, for which the structure was located at different longitudinal positions. The remarkable quantitative agreement of the residual phase aberrations (0.006 waves rms) in Fig. 4(c) demonstrates that we can accurately and consistently retrieve the wavefront aberrations. Furthermore, it demonstrates that our technique is capable of accurate wavefront measurement when the moveable structure is significantly separated from the beam focus. This capability could prove useful for "remotely" probing the quality of the focus and for characterization and alignment of nanofocusing x-ray optics.

Use of the NSLS, the CFN, and the NSLS-II project at BNL was supported by the U.S. DOE, Office of Basic Energy Sciences, under Contract No. DE-AC02-98CH10886, and the use of ANL-APS is supported through the DOE Contract No. DE-AC02-06CH11357. This work was performed in part at the Cornell CNF, a member of NNIN, which is supported by the NSF under Grant No. ECS-0335765.

${ }^{1}$ D. Malacara, Optical Shop Testing (Wiley, New Jersey, 2007).

${ }^{2}$ M. Idir, P. Mercere, M. H. Modi, G. Dovillaire, X. Levecq, S. Bucourt, L. Escolano, and P. Sauvageot, Nucl. Instrum. Methods Phys. Res. A 616, 162 (2010).

${ }^{3}$ T. Weitkamp, B. Nöhammer, A. Diaz, C. David, and E. Ziegler, Appl. Phys. Lett. 86, 054101 (2005).

${ }^{4}$ H. M. L. Faulkner and J. M. Rodenburg, Phys. Rev. Lett. 93, 023903 (2004).

${ }^{5}$ M. Guizar-Sicairos and J. R. Fienup, Opt. Express 17, 2670 (2009).

${ }^{6}$ C. M. Kewish, P. Thibault, M. Dierolf, O. Bunk, A. Menzel, J. VilaComamala, K. Jefimovs, and F. Pfeiffer, Ultramicroscopy 110, 325 (2010).

${ }^{7}$ A. Schropp, P. Boye, J. M. Feldkamp, R. Hoppe, J. Patommel, D. Samberg, S. Stephan, K. Giewekemeyer, R. N. Wilke, T. Salditt, J. Gulden, A P. Mancuso, I. A. Vartanyants, E. Weckert, S. Schöder, M. Burghammer, and C. G. Schroer, Appl. Phys. Lett. 96, 091102 (2010).

${ }^{8}$ M. Guizar-Sicairos, K. Evans-Lutterodt, A. F. Isakovic, A. Stein, J. B. Warren, A. R. Sandy, S. Narayanan, and J. R. Fienup, Opt. Express 18, 18374 (2010).

${ }^{9}$ C. M. Kewish, M. Guizar-Sicairos, C. Liu, J. Qian, B. Shi, C. Benson, A. M. Khounsary, J. Vila-Comamala, O. Bunk, J. R. Fienup, A. T. Macrander, and L. Assoufid, Opt. Express 18, 23420 (2010).

${ }^{10}$ H. Mimura, S. Handa, T. Kimura, H. Yumoto, D. Yamakawa, H. Yokoyama, S. Matsuyama, K. Inagaki, K. Yamamura, Y. Sano, K. Tamasaku, Y. Nishino, M. Yabashi, T. Ishikawa, and K. Yamauchi, Nat. Phys. 6, 122 (2010).

${ }^{11}$ A. F. Isakovic, K. Evans-Lutterodt, D. Elliott, A. Stein, and J. B. Warren, J. Vac. Sci. Technol. A 26, 1182 (2008).

${ }^{12}$ K. Evans-Lutterodt, A. Stein, J. M. Ablett, N. Bozovic, A. Taylor, and D. M. Tennant, Phys. Rev. Lett. 99, 134801 (2007).

${ }^{13}$ M. Guizar-Sicairos and J. R. Fienup, Opt. Express 16, 7264 (2008).

${ }^{14}$ A. E. Siegman and E. A. Sziklas, Appl. Opt. 13, 2775 (1974). 